\title{
A Discussion of Non-Constant Creep Activation Energy
}

\section{Gray $\mathbf{V}^{*}$ and Whittaker $M$}

Institute of Structural Materials, Swansea University, Fabian Way SA1 8EN, UK

\begin{abstract}
This paper explores the concept of creep activation energy, comparing the currently used Arrhenius equation to an outcome of the using Gibbs free energy. The consequence of these differing approaches is illustrated using large datasets. By examining the fundamental approach to creep activation energy, this article highlights potential advances in the field of creep in terms of activation energy, modelling, region splitting and mechanism mapping.
\end{abstract}

Keywords: Activation energy; Creep; Arrhenius; Gibbs; Region splitting

\section{Introduction}

Creep is a relatively new field of study with the first standards defined in the late 1940s and early 1950s [1-4]. The backbone of creep is the use of the Arrhenius equation to characterise the activation energy [5]. The Arrhenius equation is an empirical relationship that describes the effect of temperature on a rate constant, $\mathrm{k}$ :

$$
k=A \exp \left(-\frac{E_{a}}{R T}\right)
$$

Such that the activation energy is described by:

$$
E_{a}=-R\left(\frac{\delta \ln (k)}{\delta(1 / T)}\right)_{P}
$$

Where, $\mathrm{A}$ is a factor of the system, $\mathrm{E}_{\mathrm{a}}$ is the activation energy, $\mathrm{R}$ is the gas constant, and $\mathrm{T}$ is the temperature. This relationship was defined from empirical evidence to describe the chemical process of diffusion [5], and was applied to creep such that activation energy could be determined by the following relationships:

$$
\begin{aligned}
& Q_{c}=R\left(\frac{\delta \ln \left(t_{f}\right)}{\delta(1 / T)}\right)_{\sigma} \\
& Q_{c}=-R\left(\frac{\delta \ln \left(\dot{\varepsilon}_{\min }\right)}{\delta(1 / T)}\right)_{\sigma}
\end{aligned}
$$

Where $\mathrm{Q}_{\mathrm{c}}$ is the activation energy in $\mathrm{kJ} / \mathrm{mol}$, $\mathrm{t}_{\mathrm{f}}$ is the time to failure, $\dot{\varepsilon}_{\min }$ is the minimum creep rate, and $\sigma$ is stress in $\mathrm{MPa}$. The use of $\mathrm{t}_{\mathrm{f}}$ or $\dot{\varepsilon}_{\min }$ is not discussed here as it is considered a separate issue. This form of creep activation energy in eqn. (3) is the most commonly used [6] and forms the backbone of most creep lifing models [7].

In thermodynamic terms, the Arrhenius activation energy closely resembles that of a canonical system where the system temperature, activation volume and number of microstates are constrained. In this case, microstates refer to the individual events of each mechanism such that 'constant microstates' assumes a fixed total number of diffusing atoms and/or dislocations. Constraining a creep test to this definition is problematic in that although a test specimen is constrained by being a solid, the activation volume changes with mechanism. Thinking about diffusion, the activation volume is one atomic volume, whereas the activation volume of a dislocation is a multiplication of dislocation length, Burgers vector and distance to saddle point which is substantially greater. Therefore a change in mechanism from diffusion to dislocation would not be characterised by the canonical or Arrhenius system.

In 1961, Davies proposed using the Gibbs free energy to model a creep system and thus activation energy [8]. He described the system as isothermal-isobaric meaning constant temperature and constant pressure. In 1964 Gibbs substantiated Davies approach [9]. Taking eqn. (1) and using the Gibbs free energy as the activation energy it yields:

$$
E_{a}=k T^{2}(\partial \ln (k) / \partial T)_{\tau}=\Delta H-T(\partial \Delta H / \partial T)_{\tau}+T^{2}(\partial \Delta S / \partial T)_{\tau}
$$

Where $\mathrm{H}$ is enthalpy, $\mathrm{S}$ is entropy and $\tau$ is the shear stress of the activation event. To deal with eqn. (4) we assume the specimen is in the lowest energy state possible and that that energy state is a ground state that includes dislocations and internal stresses i.e. minimum energy for a system with flaws rather than an absolute minimum energy system. This means the second law of thermodynamics gives the following relationship:

$$
T d S=d U-\tau d V
$$

If the system is in an excited state then $S=S_{1}+\Delta S, U=U_{1}+\Delta U$ and $\mathrm{V}=\mathrm{V}_{1}+\Delta \mathrm{V}$ leading to:

$$
T d \Delta S=d \Delta U-\tau d \Delta V
$$

Implementing eqn. (6) we get:

$$
(\partial \Delta H / \partial T)_{\tau}=T(\partial \Delta S / \partial T)_{\tau}
$$

Taking eqn. (7) and substituting into eqn. (4) we get the following description of activation energy:

$$
E_{a}=\Delta H=d \Delta U-\tau d \Delta V
$$

It is at this point Gibbs concludes with defining activation energy for thermally activated glide in terms of $\Delta \mathrm{G}$ rather than $\Delta \mathrm{H}[9]$.

This approach to creep activation energy using the Gibbs free energy has wider implications than observed by Davies [8] and

${ }^{*}$ Corresponding author: Dr. V Gray, Research Officer, Institute of Structural Materials, College of Engineering, Swansea University, UK, Tel: +44 1792602061; E-mail: v.a.gray@swansea.ac.uk

Received August 17, 2017; Accepted August 31, 2017; Published September 11,2017

Citation: Gray V, Whittaker M (2017) A Discussion of Non-Constant Creep Activation Energy. J Material Sci Eng 6: 372. doi: 10.4172/2169-0022.1000372

Copyright: (c) 2017 Gray V, et al. This is an open-access article distributed under the terms of the Creative Commons Attribution License, which permits unrestricted use, distribution, and reproduction in any medium, provided the original author and source are credited. 
Gibbs [9]. Putting eqn. (8) in traditional creep notation, observing for a uniaxial test $\tau$ is proportional to $\sigma$, noting that $\Delta \mathrm{V}$ refers to the activation volume of a creep mechanism rather than specimen volume:

$$
\begin{aligned}
& Q_{c}=-R\left(\frac{\delta \ln \left(\varepsilon_{\text {min }}^{\ddot{y}}\right)}{\delta(1 / T)}\right)_{\sigma}-\sigma \Delta V \\
& Q_{c}=R\left(\frac{\delta \ln \left(t_{f}\right)}{\delta(1 / T)}\right)_{\sigma}-\sigma \Delta V
\end{aligned}
$$

Considering eqns. (3) and (9), we see a difference in the definition and behaviour of activation energies for an Arrhenius and Gibbs system respectively. For an Arrhenius system we expect constant activation energy with respect to increasing stress for each mechanism [10]. For a Gibbs system we expect an almost linear relationship between activation energy and stress for each creep mechanism. From an initial inspection of the literature, we see evidence for creep activation energy behaving according to a Gibbs system (Figure 1) [11-13].

For more substantial evidence that creep activation energy follows the Gibbs rather than Arrhenius definition, we need to consider larger datasets. Looking at 2.25Cr-1Mo steel (Grade 22) taking the collective NIMS datasheets $3 \mathrm{~b}$ and $36 \mathrm{~b}$ for tube and plate respectively, giving a total of almost 700 results are analysed. When we derive the activation energy using $\ln \left(\mathrm{t}_{\mathrm{f}}\right)$ vs. $1 / \mathrm{T}$ we see a linear decline in activation energy with respect to increasing stress.

In Figure 2 we see three different 'regions' which relate to possible changes in mechanism as described by Maruyama [14]. Within each of these regions, the activation energy declines linearly with stress acting as a Gibbs system rather than Arrhenius.

The problem in defining creep using eqn. (9) is that activation energies for mechanisms such as diffusion/dislocations are derived from simulations such as Molecular Dynamics which are calculated at close to zero stress $[15,16]$. From Figure 2 we see that the zero stress values of test data derived activation energies for each region vary widely and reach unrealistically high values. This is a common problem for creep activation energies, for example, 9Cr-1Mo steel has reported activation energies ranging from 407-1172 kJ/mol [17-19]. As such, although the linear decrease in activation energy expected from the Gibb's approach is observed, the unrealistically high activation energy values are still derived.

The Arrhenius and Gibbs activation energies are used for chemical and gaseous systems with no limitation on pressure or stress. Under these conditions it is assumed that the activation energy is a result of the absolute amount of thermal and kinetic energy introduced to the system. For a solid there is an upper limit to the amount of stress it can withstand at a specific temperature therefore introducing a

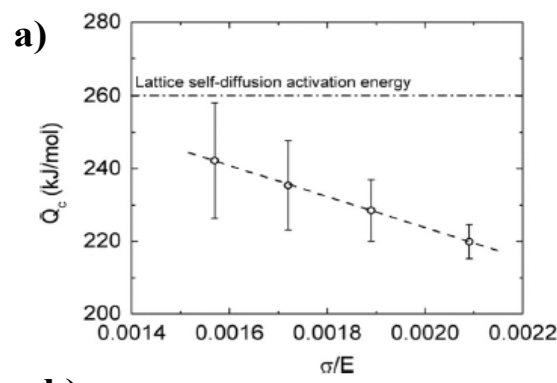

b)

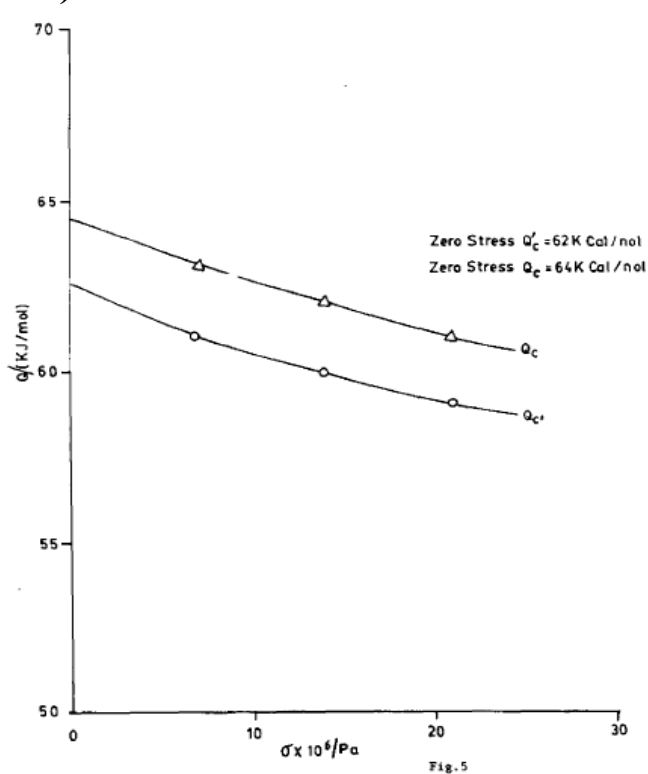

c)

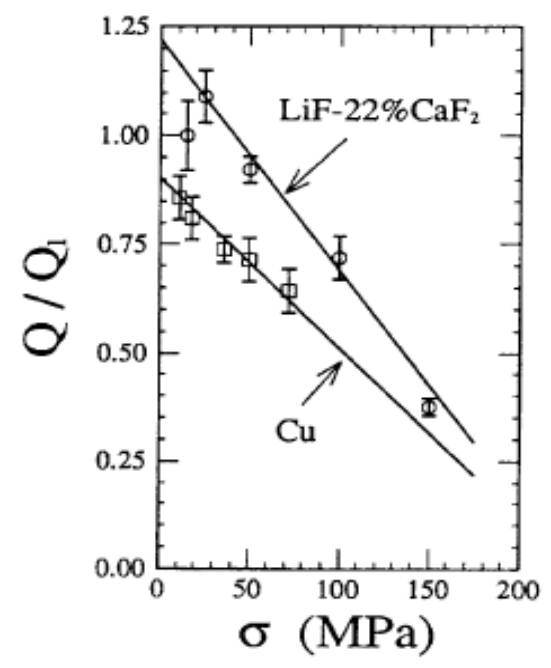

Figure 1: Creep activation energy of (a) Zirconium reproduced from [11], (b) Polycrystalline copper reproduced from ref. [12] and (c) Copper and $\mathrm{LiF}-22 \% \mathrm{CaF}_{2}$ reproduced from ref. [13]. 
limit to the system. Determining this limit would require substantial calculations for each specific system and would have variations for each melt of the same material. To approximate the kinetic/thermal limit the temperature dependent Ultimate Tensile Stress $\left(\sigma_{\mathrm{UTS}(\mathrm{T})}\right)$ or Yield Strength provide an easily obtained approximation as these values represent material properties characterising the materials reaction to kinetic/thermal energy input. In energy terms the UTS(T) closest represents the upper limit of thermal and kinetic energy the system can take before destruction which leads to the idea of evaluating creep at a normalised stress i.e. at constant $\sigma / \sigma_{\mathrm{UTS}(\mathrm{T})}[20]$. When we implement this for eqn. (9), the $\sigma \Delta \mathrm{V}$ term significantly reduces its impact as seen in Figure 3. Indeed, at zero stress the activation energies are red 280 $\mathrm{kJ} / \mathrm{mol}$, green $\sim 300 \mathrm{~kJ} / \mathrm{mol}$, and blue $\sim 315 \mathrm{~kJ} / \mathrm{mol}$ which is close to the activation energies for $\alpha$-Fe self-diffusion, and dislocation processes [21].

Looking at Figures 1 and 2, creep activation energy linearly declines with increasing stress consistent with the description of activation energy resulting from the Gibbs free energy approach and in contradiction to the Arrhenius equation. From Figures 2 and 3, we see that limiting our system to a maximum energy state through evaluation at constant normalised stress produces more realistic activation energies that are less affected by increasing stress. These outcomes are significant for the field of creep in terms of modelling, region splitting, and mechanism mapping.

When it comes to modelling creep, there are a significant number of methods. The most well-known is the power-law approach which determines the creep mechanism from a combination of Arrhenius activation energy and exponent $n$ value. Beside the issue of Arrhenius activation energy often producing high values which are unphysical, the $\mathrm{n}$ exponent is not physically based and requires significant interpretation. Arguably, if the modelling approach is accurate, then the activation energy should produce realistic values that accurately reflect the creep mechanism and as such address where and how to region split [10]. Additionally, if incorrect activation energies are inputted into creep models then the ability for that model to predict creep properties lies in its ability to compensate and cope with an incorrect assumption.

One of the great challenges of creep is the construction of a creep mechanism map. The mechanism map is a representation of the stress

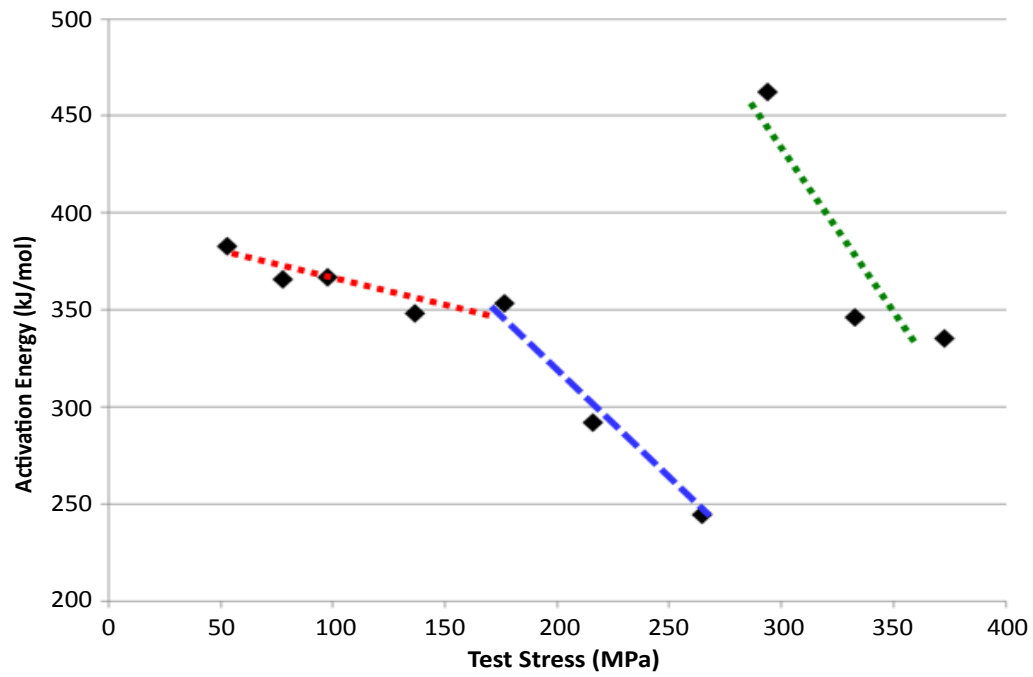

Figure 2: Activation energy of $2.25 \mathrm{Cr}-1 \mathrm{Mo}$ steel from NIMS $3 \mathrm{~b}$ and $36 \mathrm{~b}$. Red=low stress regime, blue=medium stress regime, and green=high stress regime

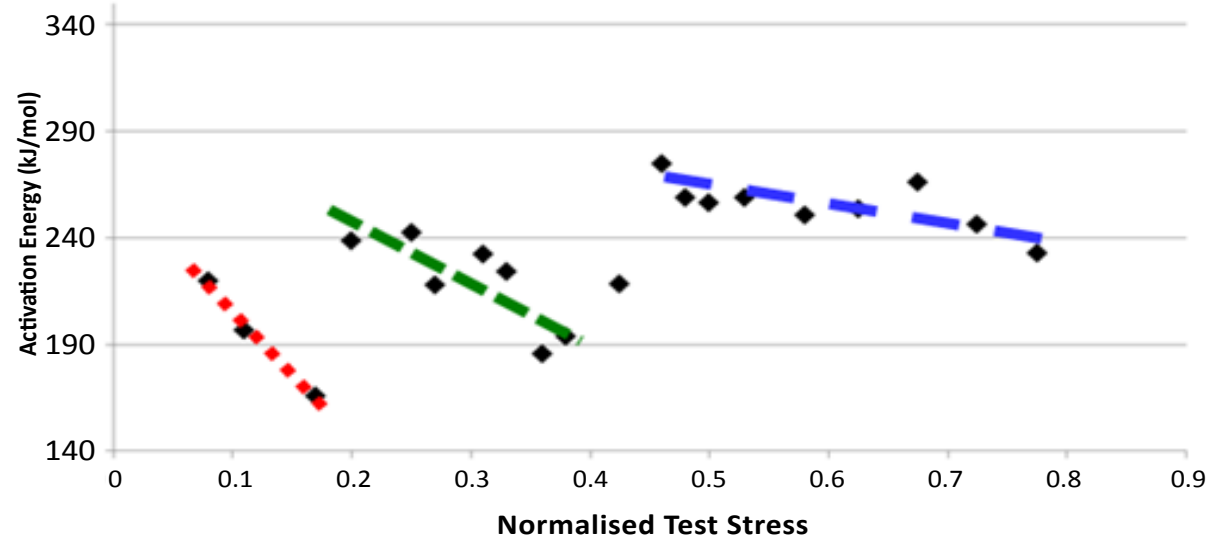

Figure 3: Normalised activation energy of $2.25 \mathrm{Cr}$-1Mo steel from NIMS $3 \mathrm{~b}$ and 36b. Normalisation interval of $\sigma / \sigma U T S(T) \pm 0.02$. Red=low stress regime, blue=medium stress regime, and green=high stress regime. 
and temperature dependent mechanisms at play in certain conditions. Currently construction of these maps relies heavily on precise creep testing, modelling, microstructural analysis, and simulation. If an accurate description of activation energy is achieved then it would be possible to construct a mechanism map from a theoretical basis using probability. For the Gibbs activation energy, the probability of a microstate/mechanism occurring is given by:

$$
P_{i}\left(Q_{c i}, V_{i}\right)=\frac{e^{-\beta\left(Q_{c i}+\sigma V_{i}\right)}}{\sum_{a} e^{-\beta\left(Q_{c a}+\sigma V_{a}\right)}}
$$

Where the probability of the creep mechanism i occurring which has activation energy $Q_{c i}$ and activation volume $V_{i}$, is given by the ratio of the activation energy of mechanism $i$ to the activation energy of all mechanisms a, noting $\beta=1 /{ }_{\mathrm{RT}}$. By having accurate activation energies of mechanisms from simulations, and, defined relationships between stress-temperature and activation volume, a mechanism map could be generated where the most probable mechanism is considered the dominant mechanism.

Creep, as a field of study faces significant challenges progressing into the $21^{\text {st }}$ century. From the power generation sector prediction of long term rupture life data from short term tests is crucial. For aerospace, there is high demand on being able to predict time to designated creep strains. The Arrhenius equation is an empirical relationship applied to creep. The Gibbs free energy approach is a physically more robust approach proposed less than 20 yrs after the advent of creep as a field of study. Given the lack of measurements at this time, the simpler Arrhenius relationship was widely taken up. Now having large datasets of multiple materials it can be seen that the Gibbs energy approach may provide a better description of creep activation energy. By physically limiting the system through evaluation at normalised stress rather than absolute stress, we have produced more realistic activation energies in a number of cases [22-24]. By having realistic activation energies, creep modelling should be able to better characterise the physical system especially in terms of region splitting where a model is partitioned based on a change in creep mechanism [10]. By having a more accurate understanding and description of the physics of creep, it is possible to foresee greater modelling accuracy, but also the ability to generate mechanism maps with fewer tests and possible greater accuracy.

\section{Acknowledgements}

This work was funded under the EPSRC Rolls-Royce Strategic Partnership in Structural Metallic Systems for Gas Turbines (grants EP/H500383/1 and EP/ $\mathrm{H} 022309 / 1)$

\section{References}

1. British Standard A23(5) (1948).

2. British Standard 1686(6) (1950).

3. British Standard 1687(7) (1950)

4. British Standard 1688(8) (1950)

5. Arrhenius S (1889) Über die Dissociationswärme und den Einfluss der Temperatur auf den Dissociationsgrad der Elektrolyte. Zeitschrift für physikalische Chemie 4: 96-116.

6. Evans RW, Wilshire B (1993) Introduction to creep. The Institute of Materials (UK), 115.

7. Abdallah Z, Gray V, Whittaker M, Perkins K (2014) A critical analysis of the conventionally employed creep lifing methods. Materials 7: 3371-3398.

8. Davies RG (1961) On the activation energy for high temperature steady state creep. Acta Metallurgica 9: 1035-1036.

9. Gibbs GB (1964) The thermodynamics of creep deformation. Physica Status Solidi (b) 5: 693-696.
10. Gray V, Whittaker M (2015) The changing constants of creep: A letter on region splitting in creep lifing. Materials Science and Engineering: A 632: 96-102.

11. Kombaiah B, Murty KL (2015) Dislocation cross-slip controlled creep in Zircaloy-4 at high stresses. Materials Science and Engineering: A 623: 114-123.

12. Ayensu A, Quainoo GK, Adjepong SK (1993) International Centre for Theoretical Physics Report IC/93/347. IAEA.

13. Freed AD, Raj SV, Walker KP (1991) Stress versus temperature dependent activation energies in creep. NASA Technical Report: TM-103192.

14. Maruyama K, Armaki HG, Yoshimi K (2007) Multiregion analysis of creep rupture data of 316 stainless steel. International Journal of Pressure Vessels and Piping 84: 171-176.

15. Attig N, Binder K, Grubmuller H, Kremer K (2004) Computational soft matter from synthetic polymers to proteins. John von Neumann Institute for Computing (NIC), Juelich.

16. Marx D, Hutter J (2000) Ab initio molecular dynamics: Theory and implementation. Modern Methods and Algorithms of Quantum Chemistry 1: 301-449.

17. Shrestha T, Basirat M, Charit I, Potirniche GP, Rink KK (2013) Creep rupture behavior of Grade 91 steel. Materials Science and Engineering: A 565: 382391.

18. Choudhary BK, Samuel EI (2011) Creep behaviour of modified $9 \mathrm{Cr}-1 \mathrm{Mo}$ ferritic steel. Journal of Nuclear Materials 412: 82-89.

19. Potirniche G, Barlow FD, Charit I, Rink K (2013) Prediction and Monitoring Systems of Creep-Fracture Behavior of $9 \mathrm{Cr}-1$ Mo Steels for Teactor Pressure Vessels (No. DOE/NEUP--09-835). Univ. of Idaho, Moscow ID (United States).

20. Williams SJ, Bache MR, Wilshire B (2010) Recent developments in the analysis of high temperature creep and creep fracture behaviour. Materials Science \& Technology 26:1332-1337.

21. Berns H, Broeckmann C, Hinz HF (2002) Creep of high speed steels Part Iexperimental investigations. Interaction 2: 4.

22. Whittaker MT, Harrison WJ, Lancaster RJ, Williams S (2013) An analysis of modern creep lifing methodologies in the titanium alloy Ti6-4. Materials Science and Engineering: A 577: 114-119.

23. Whittaker MT, Evans M, Wilshire B (2012) Long-term creep data prediction for type $316 \mathrm{H}$ stainless steel. Materials Science and Engineering: A 552: 145-150.

24. Whittaker MT, Wilshire B (2013) Advanced procedures for long-term creep data prediction for 2.25 chromium steels. Metallurgical and Materials Transactions A 44: 136-153. 